\title{
SOME APPLICATIONS OF GENERALIZED SRIVASTAVA-ATTIYA OPERATOR TO THE BI-CONCAVE FUNCTIONS
}

\author{
ŞAHSENE ALTINKAYA AND SIBEL YALÇIN
}

Received 25 April, 2019

\begin{abstract}
In this present investigation, we are concerned with the class $\Omega_{\Sigma ; \mu, b}^{m, k} C_{0}(\alpha)$ of bi-concave functions defined by using the generalized Srivastava-Attiya operator. Moreover, we derive some coefficient inequalities for functions in this class.
\end{abstract}

2010 Mathematics Subject Classification: 30C45

Keywords: bi-concave functions, convolution, Srivastava-Attiya operator.

\section{INTRODUCTION}

Let $A$ indicate an analytic function family, which is normalized under the condition of $f(0)=f^{\prime}(0)-1=0$ in $\mathbb{D}=\{z: z \in \mathbb{C}$ and $|z|<1\}$ and given by the following Taylor-Maclaurin series:

$$
f(z)=z+\sum_{n=2}^{\infty} a_{n} z^{n}
$$

Further, by $S$ we shall denote the class of all functions in $A$ which are univalent in $\mathbb{D}$.

It is well known that every function $f \in S$ has an inverse $f^{-1}$, defined by $f^{-1}(f(z))=z,(z \in \mathbb{D})$ and $f\left(f^{-1}(w)\right)=w,\left(|w|<r_{0}(f) ; r_{0}(f) \geq \frac{1}{4}\right)$. In fact, the inverse function is given by

$$
f^{-1}(w)=w-a_{2} w^{2}+\left(2 a_{2}^{2}-a_{3}\right) w^{3}-\left(5 a_{2}^{3}-5 a_{2} a_{3}+a_{4}\right) w^{4}+\cdots
$$

(for details, see Duren [13]). A function $f \in A$ is said to be bi-univalent in $\mathbb{D}$ if both $f$ and $f^{-1}$ are univalent in $\mathbb{D}$. Let $\Sigma$ stand for the class of bi-univalent functions defined in the unit disk $\mathbb{D}$. A brief history and interesting examples of functions in the class $\Sigma$ can be found in the pioneering work on this subject by Srivastava et al. [34], which has apparently revived the study of bi-univalent functions in recent years. In fact, ever since the publication by Srivastava et al. [34], a huge flood of papers have appeared and are still appearing in the literature dealing with various subclasses of the bi-univalent and other related function classes (see, for example, $[6,7,20,24,26,32,35,37])$. But the coefficient problem for each one of the following 
Taylor-Maclaurin coefficients

$$
\left|a_{n}\right|, n \in \mathbb{N} \backslash\{1,2\} ; \mathbb{N}=\{1,2,3, \ldots\}
$$

is still an open problem. In the literature, there are only a few works determining the general coefficient bounds $\left|a_{n}\right|$ for the analytic bi-univalent functions ([11,16,33,36, 38]).

The study of operators plays an important role in Geometric Function Theory in Complex Analysis and its related fields. Recently, the interest in this area has been increasing because it permits detailed investigations of problems with physical applications. For example, many derivative and integral operators can be written in terms of convolution of certain analytic functions. For functions

$$
f_{j}(z)=\sum_{n=0}^{\infty} a_{n, j} z^{n} \quad(j=1,2)
$$

analytic in $\mathbb{D}$, we define the Hadamard product of $f_{1}$ and $f_{2}$ as

$$
\left(f_{1} * f_{2}\right)(z)=\sum_{n=0}^{\infty} a_{n, 1} a_{n, 2} z^{n}=\left(f_{2} * f_{1}\right)(z) \quad(z \in \mathbb{D}) .
$$

In terms of the Hadamard product (or convolution), the Dziok-Srivastava linear convolution operator involving the generalized hypergeometric function was introduced and studied systematically by Dziok and Srivastava $[14,15]$ and (subsequently) by many other authors (see, for details, [17, 18, 29]). We recall here a general HurwitzLerch Zeta function $\Phi(z, s, a)$ defined in [31] by

$$
\Phi(z, s, a):=\sum_{n=0}^{\infty} \frac{z^{n}}{(n+a)^{s}}
$$

$\left(a \in \mathbb{C} \backslash \mathbb{Z}_{0}^{-} ; s \in \mathbb{C}\right.$, when $|z|<1 ; \Re(s)>1$ when $\left.|z|=1\right)$, where $\mathbb{Z}_{0}^{-}:=\mathbb{Z} \backslash \mathbb{N}$. Several interesting properties and characteristics of the Hurwitz-Lerch Zeta function $\Phi(z, s, a)$ can be found in [9], and the references stated there in (see also [20], [21, 30]). Srivastava and Attiya [30] (also see [2,8,19]) introduced and investigated the linear operator:

$$
\Omega_{b}^{\mu}: A \rightarrow A
$$

defined in terms of the Hadamard product by

$$
\Omega_{b}^{\mu} f(z)=\left(G_{b}^{\mu} * f\right)(z), \quad\left(z \in \mathbb{D} ; b \in \mathbb{C} \backslash \mathbb{Z}_{0}^{-} ; \mu \in \mathbb{C} ; f \in A\right),
$$

where, for convenience,

$$
G_{b}^{\mu}(z):=(1+b)^{\mu}\left[\Phi(z, \mu, b)-b^{-\mu}\right] \quad(z \in \mathbb{D}) .
$$

Next, we recall the following relationships which follow easily by using (1.1), (1.3) and (1.4)

$$
\Omega_{b}^{\mu} f(z)=z+\sum_{n=2}^{\infty}\left(\frac{1+b}{n+b}\right)^{\mu} a_{n} z^{n} .
$$


Motivated essentially by the Srivastava-Attiya operator, Murugusundaramoorthy [25] introduced the generalized integral operator $\Omega_{\mu, b}^{m, k}$ given by

$$
\Omega_{\mu, b}^{m, k} f(z)=z+\sum_{n=2}^{\infty} C_{n}^{m}(b, \mu, k) a_{n} z^{n}
$$

where

$$
C_{n}^{m}(b, \mu, k)=\left|\left(\frac{1+b}{n+b}\right)^{\mu}\right| \frac{m !(n+k-2) !}{(k-2) !(n+m-1) !}
$$

and (throughout this paper unless otherwise mentioned) the parameters $\mu, b$ are constrained as $b \in \mathbb{C} \backslash \mathbb{Z}_{0}^{-} ; \mu \in \mathbb{C}, k \geq 2$ and $m>-1$. It is of interest to note that $\Omega_{\mu, b}^{1,2}$ is the Srivastava-Attiya operator and $\Omega_{0, b}^{m, k}$ is the well-known Choi-Saigo-Srivastava operator (see [22]). Suitably specializing the parameters $m, k, \mu$ and $b$ in $\Omega_{\mu, b}^{m, k} f(z)$, we can get various integral operators introduced by Alexander [1] and Bernardi [4], Ling and Liu [22], Livingstone [23].

\section{PRELIMINARIES}

Conformal maps of the unit disk onto convex domains are a classical topic. Recently Avkhadiev and Wirths [3] discovered that conformal maps onto concave domains (the complements of convex closed sets) have some novel properties.

A function $f: \mathbb{D} \rightarrow \mathbb{C}$ is said to belong to the family $C_{0}(\alpha)$ if $f$ satisfies the following conditions:

- $f$ is analytic in $\mathbb{D}$ with the standard normalization $f(0)=f^{\prime}(0)-1=0$. In addition it satisfies $f(1)=\infty$,

- $f$ maps $\mathbb{D}$ conformally onto a set whose complement with respect to $\mathbb{C}$ is convex,

- the opening angle of $f(\mathbb{D})$ at $\infty$ is less than or equal to $\pi \alpha, \alpha \in(1,2]$.

The class $C_{0}(\alpha)$ is referred to as the class of concave univalent functions and for a detailed discussion about concave functions, we refer to Avkhadiev and Wirths [3], Cruz and Pommerenke [10] and references there in.

In particular, the inequality

$$
\Re\left(1+\frac{z f^{\prime \prime}(z)}{f^{\prime}(z)}\right)<0 \quad(z \in \mathbb{D})
$$

is used - sometimes also as a definition - for concave functions $f \in C_{0_{o}}$ (see e.g. [27] and others).

Bhowmik et al. [5] showed that an analytic function $f$ maps $\mathbb{D}$ onto a concave domain of angle $\pi \alpha$, if and only if $\Re\left(P_{f}(z)\right)>0$, where

$$
P_{f}(z)=\frac{2}{\alpha-1}\left[\frac{\alpha+1}{2} \frac{1+z}{1-z}-1-z \frac{f^{\prime \prime}(z)}{f^{\prime}(z)}\right] .
$$


There has been a number of investigations on basic subclasses of concave univalent functions (see, for example [12], [28]).

To establish our new subclass we require the following Definition.

Definition 1. Let the functions $h, p: \mathbb{D} \rightarrow \mathbb{C}$ be so constrained that

$$
\min \{\Re(h(z)), \mathfrak{R}(p(z))\}>0
$$

and

$$
h(0)=p(0)=1 .
$$

Motivated by each of the above definitions, we now define a new subclass of biconcave analytic functions involving the generalized integral operator $\Omega_{\mu, b}^{m, k}$.

Definition 2. A function $f \in \Sigma$ given by (1.1) is said to be in the class

$$
\Omega_{\Sigma ; \mu, b}^{m, k} C_{0}(\alpha) \quad\left(b \in \mathbb{C} \backslash \mathbb{Z}_{0}^{-} ; \mu \in \mathbb{C} ; k \geq 2 ; m>-1 ; \alpha \in(1,2] ; z, w \in \mathbb{D}\right)
$$

the following conditions are satisfied:

$$
\frac{2}{\alpha-1}\left[\frac{\alpha+1}{2} \frac{1+z}{1-z}-1-z \frac{\left[\Omega_{\Sigma ; \mu, b}^{m, k} f(z)\right]^{\prime \prime}}{\left[\Omega_{\Sigma ; \mu, b}^{m, k} f(z)\right]^{\prime}}\right] \in h(\mathbb{D})
$$

and

$$
\frac{2}{\alpha-1}\left[\frac{\alpha+1}{2} \frac{1-w}{1+w}-1-w \frac{\left[\Omega_{\Sigma ; \mu, b}^{m, k} g(w)\right]^{\prime \prime}}{\left[\Omega_{\Sigma ; \mu, b}^{m, k} g(w)\right]^{\prime}}\right] \in p(\mathbb{D}),
$$

where $g(w)=f^{-1}(w)$.

\section{MAin Results AND THEIR CONSEQUences}

We begin by finding the estimates on the coefficients $\left|a_{2}\right|$ and $\left|a_{3}\right|$ for functions in the class $\Omega_{\Sigma ; \mu, b}^{m, k} C_{0}(\alpha)$.

Theorem 1. Let $f$ given by (1.1) be in the class $\Omega_{\Sigma ; \mu, b}^{m, k} C_{0}(\alpha)$. Then

$$
\begin{gathered}
\left|a_{2}\right| \leq \min \left\{\sqrt{\frac{8(\alpha+1)^{2}+(\alpha-1)^{2}\left(\left|h^{\prime}(0)\right|^{2}+\left|p^{\prime}(0)\right|^{2}\right)}{32\left[C_{2}^{m}(b, \mu, k)\right]^{2}}+\frac{\left(\alpha^{2}-1\right)\left(\left|h^{\prime}(0)\right|+\left|p^{\prime}(0)\right|\right)}{8\left[C_{2}^{m}(b, \mu, k)\right]^{2}}},\right. \\
\left.\sqrt{\frac{(\alpha+1)}{2\left|2\left[C_{2}^{m}(b, \mu, k)\right]^{2}-3 C_{3}^{m}(b, \mu, k)\right|}+\frac{(\alpha-1)\left(\left|h^{\prime \prime}(0)\right|+\left|p^{\prime \prime}(0)\right|\right)}{16\left|2\left[C_{2}^{m}(b, \mu, k)\right]^{2}-3 C_{3}^{m}(b, \mu, k)\right|}}\right\}
\end{gathered}
$$

and

$$
\left|a_{3}\right| \leq \min \left\{\frac{8(\alpha+1)^{2}+(\alpha-1)^{2}\left(\left|h^{\prime}(0)\right|^{2}+\left|p^{\prime}(0)\right|^{2}\right)+4\left(\alpha^{2}-1\right)\left(\left|h^{\prime}(0)\right|+\left|p^{\prime}(0)\right|\right)}{32\left[C_{2}^{m}(b, \mu, k)\right]^{2}}\right.
$$




$$
\begin{gathered}
+\frac{(\alpha-1)\left(\left|h^{\prime \prime}(0)\right|+\left|p^{\prime \prime}(0)\right|\right)}{48 C_{3}^{m}(b, \mu, k)}, \\
\left.\frac{(\alpha-1)\left\{\left|3 C_{3}^{m}(b, \mu, k)-\left[C_{2}^{m}(b, \mu, k)\right]^{2}\right|\left|h^{\prime \prime}(0)\right|+\left[C_{2}^{m}(b, \mu, k)\right]^{2}\left|p^{\prime \prime}(0)\right|\right\}+12(\alpha+1) C_{3}^{m}(b, \mu, k)}{24 C_{3}^{m}(b, \mu, k)\left|2\left[C_{2}^{m}(b, \mu, k)\right]^{2}-3 C_{3}^{m}(b, \mu, k)\right|}\right\} .
\end{gathered}
$$

Proof. Let $f \in \Omega_{\Sigma ; \mu, b}^{m, k} C_{0}(\alpha)$ and $g$ be the analytic extension of $f^{-1}$ to $\mathbb{D}$. It follows from (2.1) and (2.2) that

$$
\frac{2}{\alpha-1}\left[\frac{\alpha+1}{2} \frac{1+z}{1-z}-1-z \frac{\left[\Omega_{\Sigma ; \mu, b}^{m, k} f(z)\right]^{\prime \prime}}{\left[\Omega_{\Sigma ; \mu, b}^{m, k} f(z)\right]^{\prime}}\right]=h(z)
$$

and

$$
\frac{2}{\alpha-1}\left[\frac{\alpha+1}{2} \frac{1-w}{1+w}-1-w \frac{\left[\Omega_{\Sigma ; \mu, b}^{m, k} g(w)\right]^{\prime \prime}}{\left[\Omega_{\Sigma ; \mu, b}^{m, k} g(w)\right]^{\prime}}\right]=p(w),
$$

where $h$ and $p$ satisfy the conditions of Definiton 1. Furthermore, the functions $h$ and $p$ have the following Taylor-Maclaurin series expensions:

$$
h(z)=1+h_{1} z+h_{2} z^{2}+\cdots
$$

and

$$
p(w)=1+p_{1} w+p_{2} w^{2}+\cdots,
$$

respectively. Now, equating the coefficients in (3.3) and (3.4), we get

$$
\begin{gathered}
\frac{2\left[(\alpha+1)-2 C_{2}^{m}(b, \mu, k) a_{2}\right]}{\alpha-1}=h_{1}, \\
\frac{2\left[(\alpha+1)-2\left(3 C_{3}^{m}(b, \mu, k) a_{3}-2\left[C_{2}^{m}(b, \mu, k)\right]^{2} a_{2}^{2}\right)\right]}{\alpha-1}=h_{2}
\end{gathered}
$$

and

$$
\begin{gathered}
-\frac{2\left[(\alpha+1)-2 C_{2}^{m}(b, \mu, k) a_{2}\right]}{\alpha-1}=p_{1}, \\
\frac{2\left[(\alpha+1)+2\left\{2\left[C_{2}^{m}(b, \mu, k)\right]^{2} a_{2}^{2}-3 C_{3}^{m}(b, \mu, k)\left(2 a_{2}^{2}-a_{3}\right)\right\}\right]}{\alpha-1}=p_{2} .
\end{gathered}
$$

From (3.5) and (3.7), we find that

$$
h_{1}=-p_{1}
$$

Also, from (3.5), we can write

$$
a_{2}=\frac{\alpha+1}{2 C_{2}^{m}(b, \mu, k)}-\frac{h_{1}(\alpha-1)}{4 C_{2}^{m}(b, \mu, k)} .
$$


Next, by using (3.5), (3.7), (3.9) and (3.10), we get

$$
a_{2}^{2}=\frac{(\alpha+1)^{2}}{4\left[C_{2}^{m}(b, \mu, k)\right]^{2}}+\frac{(\alpha-1)^{2}\left(h_{1}^{2}+p_{1}^{2}\right)}{32\left[C_{2}^{m}(b, \mu, k)\right]^{2}}-\frac{\left(\alpha^{2}-1\right)\left(h_{1}-p_{1}\right)}{8\left[C_{2}^{m}(b, \mu, k)\right]^{2}} .
$$

By adding (3.6) to (3.8), we get

$$
a_{2}^{2}=\frac{(\alpha-1)\left(h_{2}+p_{2}\right)}{8\left(2\left[C_{2}^{m}(b, \mu, k)\right]^{2}-3 C_{3}^{m}(b, \mu, k)\right)}-\frac{\alpha+1}{2\left(2\left[C_{2}^{m}(b, \mu, k)\right]^{2}-3 C_{3}^{m}(b, \mu, k)\right)} .
$$

Thus, we find from the equations (3.11) and (3.12) that

$$
\left|a_{2}\right|^{2} \leq \frac{(\alpha+1)^{2}}{4\left[C_{2}^{m}(b, \mu, k)\right]^{2}}+\frac{(\alpha-1)^{2}\left(\left|h^{\prime}(0)\right|^{2}+\left|p^{\prime}(0)\right|^{2}\right)}{32\left[C_{2}^{m}(b, \mu, k)\right]^{2}}+\frac{\left(\alpha^{2}-1\right)\left(\left|h^{\prime}(0)\right|+\left|p^{\prime}(0)\right|\right)}{8\left[C_{2}^{m}(b, \mu, k)\right]^{2}}
$$

and

$$
\left|a_{2}\right|^{2} \leq \frac{(\alpha+1)}{2\left|2\left[C_{2}^{m}(b, \mu, k)\right]^{2}-3 C_{3}^{m}(b, \mu, k)\right|}+\frac{(\alpha-1)\left(\left|h^{\prime \prime}(0)\right|+\left|p^{\prime \prime}(0)\right|\right)}{16\left|2\left[C_{2}^{m}(b, \mu, k)\right]^{2}-3 C_{3}^{m}(b, \mu, k)\right|} .
$$

Similarly, subtracting (3.8) from (3.6), we have

$$
a_{3}=a_{2}^{2}-\frac{(\alpha-1)\left(h_{2}-p_{2}\right)}{24 C_{3}^{m}(b, \mu, k)} .
$$

Then, upon substituting the value of $a_{2}^{2}$ from (3.11) and (3.12) into (3.13), we deduce that

$$
\begin{gathered}
a_{3}= \\
\frac{(\alpha+1)^{2}}{4\left[C_{2}^{m}(b, \mu, k)\right]^{2}}+\frac{(\alpha-1)^{2}\left(h_{1}^{2}+p_{1}^{2}\right)}{32\left[C_{2}^{m}(b, \mu, k)\right]^{2}}-\frac{\left(\alpha^{2}-1\right)\left(h_{1}-p_{1}\right)}{8\left[C_{2}^{m}(b, \mu, k)\right]^{2}}-\frac{(\alpha-1)\left(h_{2}-p_{2}\right)}{24 C_{3}^{m}(b, \mu, k)} \\
=\frac{(\alpha-1)\left(h_{2}+p_{2}\right)}{8\left(2\left[C_{2}^{m}(b, \mu, k)\right]^{2}-3 C_{3}^{m}(b, \mu, k)\right)}-\frac{\alpha+1}{2\left(2\left[C_{2}^{m}(b, \mu, k)\right]^{2}-3 C_{3}^{m}(b, \mu, k)\right)}-\frac{(\alpha-1)\left(h_{2}-p_{2}\right)}{24 C_{3}^{m}(b, \mu, k)} .
\end{gathered}
$$

Consequently, we have

$$
\left|a_{3}\right| \leq \frac{8(\alpha+1)^{2}+(\alpha-1)^{2}\left(\left|h^{\prime}(0)\right|^{2}+\left|p^{\prime}(0)\right|^{2}\right)+4\left(\alpha^{2}-1\right)\left(\left|h^{\prime}(0)\right|+\left|p^{\prime}(0)\right|\right)}{32\left[C_{2}^{m}(b, \mu, k)\right]^{2}}+\frac{(\alpha-1)\left(\left|h^{\prime \prime}(0)\right|+\left|p^{\prime \prime}(0)\right|\right)}{48 C_{3}^{m}(b, \mu, k)}
$$

and

$$
\left|a_{3}\right| \leq \frac{(\alpha-1)\left\{\left|3 C_{3}^{m}(b, \mu, k)-\left[C_{2}^{m}(b, \mu, k)\right]^{2}\right| h^{\prime \prime}(0)\left|+\left[C_{2}^{m}(b, \mu, k)\right]^{2}\right| p^{\prime \prime}(0) \mid\right\}+12(\alpha+1) C_{3}^{m}(b, \mu, k)}{24 C_{3}^{m}(b, \mu, k)\left|2\left[C_{2}^{m}(b, \mu, k)\right]^{2}-3 C_{3}^{m}(b, \mu, k)\right|} .
$$

This completes the proof. 


\section{CONCLUSions}

It is easily seen that, by specializing the functions $h(z)$ and $p(z)$ involved in Theorem 1, some results for could be expressed as illustrative examples:

Corollary 1. If we set

$$
h(z)=\left(\frac{1+z}{1-z}\right)^{\gamma}=1+2 \gamma z+2 \gamma^{2} z^{2}+\ldots \quad(0<\gamma \leq 1),
$$

and

$$
p(z)=\left(\frac{1-z}{1+z}\right)^{\gamma}=1-2 \gamma z+2 \gamma^{2} z^{2}+\ldots \quad(0<\gamma \leq 1)
$$

then inequalities (3.1) and (3.2) become

$$
\left|a_{2}\right| \leq \min \left\{\frac{(\alpha+1)+(\alpha-1) \gamma}{2 C_{2}^{m}(b, \mu, k)}, \sqrt{\frac{(\alpha-1) \gamma^{2}+(\alpha+1)}{2\left|2\left[C_{2}^{m}(b, \mu, k)\right]^{2}-3 C_{3}^{m}(b, \mu, k)\right|}}\right\}
$$

and

$$
\begin{aligned}
\left|a_{3}\right| \leq & \min \left\{\frac{(\alpha+1)^{2}+(\alpha-1)^{2} \gamma^{2}+2\left(\alpha^{2}-1\right) \gamma}{4\left[C_{2}^{m}(b, \mu, k)\right]^{2}}+\frac{(\alpha-1) \gamma^{2}}{6 C_{3}^{m}(b, \mu, k)},\right. \\
& \left.\frac{(\alpha-1)\left\{\left|3 C_{3}^{m}(b, \mu, k)-\left[C_{2}^{m}(b, \mu, k)\right]^{2}\right| \gamma^{2}+\left[C_{2}^{m}(b, \mu, k)\right]^{2} \gamma^{2}\right\}+3(\alpha+1) C_{3}^{m}(b, \mu, k)}{6 C_{3}^{m}(b, \mu, k)\left|2\left[C_{2}^{m}(b, \mu, k)\right]^{2}-3 C_{3}^{m}(b, \mu, k)\right|}\right\} .
\end{aligned}
$$

Corollary 2. If we let

$$
h(z)=\frac{1+(1-2 \beta) z}{1-z}=1+2(1-\beta) z+2(1-\beta) z^{2}+\cdots \quad(0 \leq \beta<1),
$$

and

$$
p(z)=\frac{1-(1-2 \beta) z}{1+z}=1-2(1-\beta) z+2(1-\beta) z^{2}+\cdots \quad(0 \leq \beta<1),
$$

then inequalities (3.1) and (3.2) become

$$
\left|a_{2}\right| \leq \min \left\{\frac{(\alpha+1)+(\alpha-1)(1-\beta)}{2 C_{2}^{m}(b, \mu, k)}, \sqrt{\frac{(\alpha-1)(1-\beta)+(\alpha+1)}{2\left|2\left[C_{2}^{m}(b, \mu, k)\right]^{2}-3 C_{3}^{m}(b, \mu, k)\right|}}\right\}
$$

and

$$
\begin{aligned}
\left|a_{3}\right| \leq \min \left\{\frac{(\alpha+1)^{2}+(\alpha-1)^{2}(1-\beta)^{2}+2\left(\alpha^{2}-1\right)(1-\beta)}{4\left[C_{2}^{m}(b, \mu, k)\right]^{2}}+\frac{(\alpha-1)(1-\beta)}{6 C_{3}^{m}(b, \mu, k)},\right. \\
\\
\left.\frac{(\alpha-1)\left\{\left|3 C_{3}^{m}(b, \mu, k)-\left[C_{2}^{m}(b, \mu, k)\right]^{2}\right|(1-\beta)+\left[C_{2}^{m}(b, \mu, k)\right]^{2}(1-\beta)\right\}+3(\alpha+1) C_{3}^{m}(b, \mu, k)}{6 C_{3}^{m}(b, \mu, k)\left|2\left[C_{2}^{m}(b, \mu, k)\right]^{2}-3 C_{3}^{m}(b, \mu, k)\right|}\right\} .
\end{aligned}
$$




\section{REFERENCES}

[1] J. W. Alexander, "Functions which map the interior of the unit circle upon simple regions." Ann. of Math., vol. 17, no. 1, pp. 12-22, 1915.

[2] A. A. Attiya and A. H. Hakami, "Some subordination results associated with generalized Srivastava-Attiya operator." Adv. Difference Equ., vol. 105, pp. 1-14, 2013.

[3] F. G. Avkhadiev and K. J. Wirths, "Convex holes produce lower bounds for coefficients." Complex Variables, Theory and Application, vol. 47, pp. 556-563, 2002.

[4] S. D. Bernardi, "Convex and starlike univalent functions." Trans. Amer. Math. Soc., vol. 135, pp. 429-446, 1969.

[5] B. Bhowmik, S. Ponnusamy, and K. J. Wirths, "Characterization and the pre-Schwarzian norm estimate for concave univalent functions." Monatsh Math., vol. 161, pp. 59-75, 2010.

[6] D. A. Brannan and J. G. Clunie, Aspects of comtemporary complex analysis. New York: Proceedings of the NATO Advanced Study Instute Held at University of Durham, 1980.

[7] D. A. Brannan and T. S. Taha, "On some classes of bi-univalent functions." Studia Universitatis Babeş-Bolyai Mathematica, vol. 31, no. 2, pp. 70-77, 1986.

[8] R. Bucur, L. Andrei, and D. Breaz, "Coefficient Bounds and Fekete-Szegö Problem for a Class of Analytic Functions Defined by Using a New Differential Operator." Monatsh Math., vol. 9, pp. $1355-1368,2015$.

[9] J. Choi and H. M. Srivastava, "Certain families of series associated with the Hurwitz-Lerch Zeta function.” Appl. Math. Comput., vol. 170, pp. 399-409, 2005.

[10] L. Cruz and C. Pommerenke, "On concave univalent functions." Complex Var. Elliptic Equ., vol. 52, pp. 53-59, 2007.

[11] Ş. Altınkaya and S. Yalçın, "Coefficient bounds for a subclass of bi-univalent functions." TWMS J. Pure Appl. Math., vol. 6, no. 2, pp. 180-185, 2015.

[12] Ş. Altınkaya and S. Yalçın, "General Properties of Multivalent Concave Functions Involving Linear Operator of Carlson-Shaffer Type." Comptes rendus de l'Académie bulgare des Sciences, vol. 69, pp. 1533-1540, 2016.

[13] P. L. Duren, Univalent Functions. New York: Grundlehren der Mathematischen Wissenschaften, Springer, 1983.

[14] J. Dziok and H. M. Srivastava, "Classes of analytic functions associated with the generalized hypergeometric function.” Appl. Math. Comput., vol. 103, pp. 1-13, 1999.

[15] J. Dziok and H. M. Srivastava, "Certain subclasses of analytic functions associated with the generalized hypergeometric function.” Integral Transforms Spec. Funct., vol. 14, pp. 7-18, 2003.

[16] S. G. Hamidi and J. M. Jahangiri, "Faber polynomial coefficient estimates for analytic biclose-to-convex functions." C. R. Acad. Sci. Paris, Ser.I, vol. 352, pp. 17-20, 2014, doi: 10.1016/j.crma.2013.11.005.

[17] P. N. Kamble, M. G. Shrigan, and H. M. Srivastava, "A novel subclass of univalent functions involving operators of fractional calculus." Internat. J. Appl. Math., vol. 30, pp. 501-514, 2017.

[18] V. Kiryakova, "Criteria for univalence of the Dziok-Srivastava and the Srivastava-Owa operators in the class A." Appl. Math. Comput., vol. 218, pp. 883-892, 2011.

[19] M. A. Kutbi and A. A. Attiya, "Differential subordination results for certain integrodifferential operator and its applications." Abst. Appl. Anal., vol. 2012, pp. 1-13, 2012.

[20] M. Lewin, "On a coefficient problem for bi-univalent functions." Proc. Amer. Math. Soc., vol. 18, no. 1, pp. 63-68, 1967, doi: 10.2307/2035225.

[21] S. D. Lin, H. M. Srivastava, and P. Y. Wang, "Some espansion formulas for a class of generalized Hurwitz-Lerch Zeta functions.” Integral Transform. Spec. Funct., vol. 17, pp. 817-1827, 2006.

[22] Y. Ling and F. S. Liu, "The Choi-Saigo-Srivastava integral operator and a class of analytic functions.” Appl. Math. Comput., vol. 165, pp. 613-621, 2005. 
[23] A. E. Livingston, "On the radius of univalence of certain analytic functions." Proc. Amer. Math. Soc., vol. 17, pp. 352-357, 1966.

[24] S. Mahmood, H. M. Srivastava, N. Khan, Q. Z. Ahmad, B. Khan, and I. Ali, "Upper bound of the third Hankel determinant for a subclass of q-starlike functions." Symmetry, vol. 11, pp. 1-13, 2019, doi: doi:10.3390/sym11030347.

[25] G. Murugusundaramoorthy, "Coefficient estimate of bi-Bazilevic function defined by SrivastavaAttiya operator." Le Matematiche, vol. 69, pp. 45-56, 2014.

[26] E. Netanyahu, "The minimal distance of the image boundary from the origin and the second coefficient of a univalent function in $|z|<1$," Archive for Rational Mechanics and Analysis, vol. 32, pp. 100-112, 1969.

[27] J. Pfaltzgra and B. Pinchuk, "A variational method for classes of meromorphic functions." J. Analyse Math., vol. 24, pp. 101-150, 1971.

[28] F. M. Sakar and H. O. Güney, "Coefficient estimates for bi-concave functions." Commun. Fac. Sci. Univ. Ank. Ser. Al Math. Stat., vol. 68, no. 1, pp. 53-60, 2019.

[29] H. M. Srivastava, "Some Fox-Wright generalized hypergeometric functions and associated families of convolution operators." Appl. Anal. Discrete Math., vol. 1, pp. 56-71, 2007.

[30] H. M. Srivastava and A. A. Attiya, "An integral operator associated with the Hurwitz-Lerch Zeta function and differential subordination." Integral Transform. Spec. Funct., vol. 18, pp. 207-216, 2007.

[31] H. M. Srivastava and J. Choi, Series associated with the Zeta and related functions. Boston, Mass. USA: Kluwer Academic, 2001.

[32] H. M. Srivastava, S. Gaboury, and F. Ghanim, "Coefficient estimates for some general subclasses of analytic and bi-univalent functions." Afrika Matematika, vol. 28, no. 5-6, pp. 693-706, 2017.

[33] H. M. Srivastava, S. Khan, Q. Z. Ahmad, N. Khan, and S. Hussain, "The Faber polynomial expansion method and its application to the general coefficient problem for some subclasses of biunivalent functions associated with a certain q-integral operator." Stud. Univ. Babeş-Bolyai Math., vol. 63, no. 4, p. 419-436, 2018, doi: 10.24193/subbmath.2018.4.01.

[34] H. M. Srivastava, A. K. Mishra, and P. Gochhayat, "Certain subclasses of analytic and bi-univalent functions.” Appl. Math. Lett., vol. 23, pp. 1188-1192, 2010, doi: 10.1016/j.aml.2010.05.009.

[35] H. M. Srivastava, F. M. Sakar, and H. O. Güney, "Some general coefficient estimates for a new class of analytic and bi-univalent functions defined by a linear combination." Filomat, vol. 32, no. 4, pp. 1313-1322, 2018.

[36] H. M. Srivastava, S. Sümer Eker, S. G. Hamidi, and J. M. Jahangiri, "Faber polynomial coefficient estimates for bi-univalent functions defined by the Tremblay fractional derivative operator." Bull. Iran. Math. Soc., vol. 44, no. 1, p. 149-157, 2018, doi: 10.1007/s41980-018-0011-3.

[37] Q. H. Xu, Y. C. Gui, and H. M. Srivastava, "Coefficient estimates for a certain subclass of analytic and bi-univalent functions." Appl. Math. Lett., vol. 25, pp. 990-994, 2012.

[38] A. Zireh, E. A. Adegani, and S. Bulut, "Faber polynomial coefficient estimates for a comprehensive subclass of analytic bi-univalent functions defined by subordination." Bull. Belg. Math. Soc. Simon Stevin, vol. 23, pp. 487-504, 2016.

Authors' addresses

Şahsene Altınkaya

Bursa Uludag University, Department of Mathematics, 16059, Bursa, Turkey

E-mail address: sahsenealtinkaya@gmail.com 
Sibel Yalçın

Bursa Uludag University, Department of Mathematics, 16059 Bursa, Turkey

E-mail address: syalcin@uludag.edu.tr 